\title{
SUMMARY OF PIVOTAL TECHNIQUE OF COTTON-HARVEST ROBOT
}

\author{
Ling Wang ${ }^{1}$, Changying $\mathrm{Ji}^{1{ }^{1, *}}$ \\ ${ }^{1}$ College of Engineering, Nanjing Agricultural University, Nanjing, 210031, China \\ * Corresponding author, Address: Department of Agricultural Mechanization, College of \\ Engineering, Nanjing Agricultural University, 40 Dianjiangtai Road, Nanjing, 210031, \\ P.R. China,Tel: 13951994628, Email: chyji@njau.edu.cn
}

\begin{abstract}
Pivotal techniques of cotton-harvest robots were summarized, including image segmentation, features generation, artificial classifiers and performances evaluation. Solutions based on machine vision and pattern recognition were analyzed to distinguish ripe from under-ripe/over-ripe cottons, and rank cottons according to government grading standards.
\end{abstract}

Keywords: Cotton, Harvest robot, Classifier

\section{INTRODUCTION}

Cotton production systems have benefited from precision agriculture (PA) (Searcy et al., 2000). Agricultural robot combines advantages of Cottonharvest in manual and mechanism way, including ecological benefits, needless of biological pretreatments, high-precision of classification and economic profitability. And it has potentials to resolve these conflicts in either efficiency or quality: America is characterized by these aspects, including large scale, single-variety, middling-long fibers and one-off harvest, which are suitable for cotton harvest in mechanism way. While China is characterized by these factors, containing small-scale, multivarieties, harvest in batches, short-middling fiber, which are inapplicable in either mechanism or manual way, and the latter has been restricted by plenty of pickers (Wang et al., 2006). So cotton-harvest robot adapts to Chinese

Wang, L. and Ji, C., 2008, in IFIP International Federation for Information Processing, Volume 259; Computer and Computing Technologies in Agriculture, Vol. 2; Daoliang Li; (Boston: Springer), pp. 1459-1463. 
situation to a great extent, and cotton-harvest robot towards PA in China is a new trend in practice.

\section{PIVOTAL TECHNIQUE}

A mobile harvest robot consists of manipulators, end-effectors, mobile wheels, machine visions, control systems etc, and fundamentals in robotic auto-navigation and objects detection are obtained. Development trends of harvest robot lie in fairly accurate, nondestructive, robust and low cost, so classifiers design is the pivotal techniques.

The appearances of cottons, including size, shape, color, texture, orientation, blemishes and impurities, plays an important role of Chinese standards in the purchase of pre-harvest cottons. Various stages for classifiers design as follows, including image segmentation, features generation, artificial classifiers and performances evaluation, are interrelated and depend on the results each other to improve the overall performances.

\subsection{Image segmentation}

Intensity images segmentation algorithms are generally based on edge, threshold and region. Threshold has become a fundamental approach enjoying a significant degree of popularity, especially in applications where speed is an important factor.

Color image segmentation algorithms are also robust such as clustering and discriminant analysis based on pixels. C-means clustering segmentation algorithm is suitable for recovering compact clusters with computational simplicity (see Figure 1).
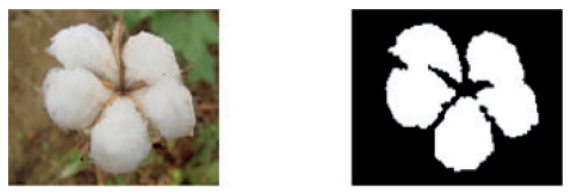

Figure 1. Segmentation of cotton based on C-means clustering

\section{$2.2 \quad$ Features generation}

Image represented as boundaries is suitable for analysis of morphology, including chain codes, moments and geometrics traditionally, and which are insensitive to position, size, orientation. Image represented as regions is used 
for evaluation of texture and color, consisting of multiple-order statistics moments, autoregressive parameters, and fractal dimension D (Pentland et al., 1984).

Features via linear transforms can exhibit high "information packing" properties. Some transforms based on problem-dependent use unfixed vectors with high computational complexity, such as Principal Component Analysis (PCA), the optimality of which leads to excellent information packing properties, and the disadvantage of which does not lead to maximum class separability in the lower dimensional subspace. Other transforms are based on fixed images and their sub-optimality with respect to decorrelation and information packing properties is often compensated by its low computational requirements, such as Fast Fourier Transform (FFT) and Discrete Wavelet Transform (DWT), and wavelet-based features selected as morphology or texture exhibit smaller within class variance and stronger between class separation than the Fourier-based ones without shift invariant (Wuncsh et al., 1995), based on which classification may result in low error rates.

\subsection{Artificial classifiers}

Classifiers are designed as follows to distinguish ripe from under/overripe cottons, and rank cottons according to government grading standards.

\subsubsection{Bayes classifier}

It classifies an unknown pattern in the most probable of the classes by minimizing the classification error probability, and assumes a known classconditional probability density functions of the feature in each class. In practice, we can assume that the features follow the general multivariate normal density due to its computational tractability and the fact that it models adequately a large number of cases.

\subsubsection{Linear discriminant}

Discriminant analysis is suboptimal with respect to Bayes classifiers by computing decision surfaces directly by means of alternative costs. Fisher linear discriminant function is an optimal linear classifier by minimizing MSE with fast, fairly accurate and easy to implement. It is useful in a wide variety of applications even if the function to be learnt is mildly nonlinear. The better generalization may be got with a simple linear classifier than with a complicated nonlinear classifier if there is too little data or too much noise. 


\subsubsection{Non-linear discriminant}

The major limitations of Support Vector Machine (SVM) are the high computational burden and the difficult selection methods of the kernel functions. A notable characteristic of SVM is suitable for high dimension features to exhibit good generalization performances.

The major limitations of Artificial Neural Network (ANN) are easy to lead the weights to a local minimum of the MSE. MSE is decomposed into the bias and the variance, and under-fitting produces excessive bias in the outputs whereas over-fitting produces excessive variance. Experiment proves that the error on training set keeps decreasing while the error on test set changes from decreasing to increasing, and the inflexion of which can be used in practice to terminate the iterations. And the size of classifiers should be kept as small as possible to a satisfactory performance.

\subsection{Performances evaluation}

Different classifiers would result in different generalization error. Resampling based cross-validation is a method for estimating generalization errors, the resulting estimates of generalization error are often used for choosing among various models. K-fold cross-validation is preferred for discontinuous error functions such as the number of misclassified cases. A value of 10 for $\mathrm{k}$ is popular for estimating generalization error while leaveone-out cross-validation often works well for estimating generalization error for continuous error functions such as MSE.

\section{CONCLUSION}

The pivotal techniques of cotton-harvest robots lie in classifiers. Machine vision and pattern recognition can be applied to recognize cottons and grade them while being harvested with a accepted accurate, and algorithms such as autoregressive model, fractal geometry, PCA, FFT, DWT, ANN, and SVM can be applied to enhance robustness of classifiers.

\section{REFERENCES}

Ling Wang, Changying Ji. Technical analysis and expectation for cotton harvesting based on agricultural robot. Cotton Science. Vol. 18(2). pp. 124-128, 2006.

Pentland A. "Fractal based decomposition of natural scenes," IEEE Transactions on Pattern Analysis and Machine Intelligence, Vol. 6(6), pp. 661-674, 1984. 
Searcy S.W., Beck A.D. Real time assessment of cotton plant height. Proceedings of Fifth International Conference on Precision Agriculture (CD), July 16 19, 2000. Bloomington, MN, USA.

Wuncsh P. Laine A. "Wavelet descriptors for multiresolution recognition of handwritten characters," Pattern Recognition, Vol. 28(8), pp. 1237-1249, 1995. 